\title{
Mining the glioma susceptibility genes in children from gene expression profiles and a methylation database
}

\author{
YONGQIANG XI $^{1 *}$, WANZHONG TANG ${ }^{2 *}$, SONG YANG $^{3}$, MAOLEI LI $^{4}$, YUCHAO HE $^{3}$ and XIANHUA FU ${ }^{3}$ \\ ${ }^{1}$ Department of Neurosurgery, The Third People's Hospital of Chengyang; ${ }^{2}$ Department of Neurosurgery, \\ Affiliated Hospital of Medical College of Qingdao University, Qingdao, Shandong 266100; \\ ${ }^{3}$ Department of Neurosurgery, Suqian First Hospital, Suqian, Jiangsu 223800; ${ }^{4}$ Department of Neurosurgery, \\ People's Hospital of Chengyang, Qingdao, Shandong 266100, P.R. China
}

Received June 19, 2015; Accepted October 24, 2016

DOI: $10.3892 / \mathrm{ol} .2017 .6579$

\begin{abstract}
Glioma is the most common type of primary brain tumor, which is associated with a poor prognosis due to its aggressive growth behavior and highly invasive nature. Research regarding glioma pathogenesis is expected to provide novel methods of adjuvant therapy for the treatment of glioma. The use of bioinformatics to identify candidate genes is commonly used to understand the genetic basis of disease. The present study used bioinformatics to mine the disease-related genes using gene expression profiles (GSE50021) and dual-channel DNA methylation data (GSE50022). The results identified 17 methylation sites located on 33 transcription factor binding sites, which may be responsible for downregulation of 17 target genes. glutamate metabotropic receptor 2 was one of the 17 downregulated target genes. Furthermore, inositol-trisphosphate 3-kinase A (ITPKA) was revealed to be the gene most associated with the risk of glioma in children. The protein coded by the ITPKA gene appeared in all risk sub-pathways, thus suggesting that ITPKA was the gene most associated with the risk of glioma, and inositol phosphate metabolism may be a key pathway associated with glioma in children. The identification of specific genes helps to determine the pathogenesis and possible therapeutic targets for the treatment of glioma in children.
\end{abstract}

\section{Introduction}

Glioma commonly occurs in the brain or spine, and is a particularly lethal solid tumor that arises from support cells in the central nervous system. Glioma can be classified into the

Correspondence to: Dr Song Yang, Department of Neurosurgery, Suqian First Hospital, 120 Suzhi Road, Sucheng, Suqian, Jiangsu 223800, P.R. China

E-mail: song20958@163.com; ysmoon@163.com

\section{${ }^{*}$ Contributed equally}

Key words: glioma, children, gene expression profile, methylation database following types: Astrocytic tumors, oligodendrogliomas and oligoastrocytomas (1). In addition, patients with glioma often suffer from fatigue, motor difficulties, drowsiness, communication difficulties, headaches and visual problems (2). A previous study reported that the median survival time of patients with the most malignant form of glioma is only 14.6 months, and the 5-year survival rate is only $9.8 \%$ (3). The high rates of morbidity and mortality associated with glioma are due to its chemoradiation resistance, and high proliferative and invasive capabilities (4).

In addition to childhood leukemia, glioma is the most common type of tumor in children, and accounts for $80 \%$ of malignant brain tumors (5). There is a peak incidence of glioma between the ages of 0 and 8 years (6). Patients with glioma that have survived surgery are more affected by their tumor and its resection than previously appreciated (7). In addition, in patients with childhood brain tumors treated with radiotherapy, memory deficits reflect general cognitive dysfunction (8). At present, the standard approach for treating glioma in children is chemotherapy; however, the safety and effectiveness of chemotherapy in children remains a problem.

Understanding the regulation of the pathological activities of glioma is important for its eradication. Research regarding glioma pathogenesis is expected to provide novel methods of adjuvant therapy for the treatment of glioma (9). With scientific improvements, the concept of epigenetics offers a potential method for cancer therapy. Epigenetics refers to stable alteration in gene expression with no underlying modifications in genetic sequence (10); DNA methylation is central to the aberrant epigentics associated with cancer (11). DNA methylation is a biochemical process where a methyl group is added to the CpG islands of DNA nucleotides by DNA methyltransferases in mammals. DNA methylation of CpG dinucleotides is considered a distinct inhibitory pathway that may mediate stable silencing (12). CpG islands are located on promoter regions, and the sequences that surround promoter regions. It has previously been reported that methylation at the $\mathrm{CpG}$ islands may induce tumorigenesis, since the abnormal expression of genes is involved in tumor development (13). Costello et al (14) reported that transcriptional repression of a tumor suppressor gene, cyclin-dependent kinase inhibitor $2 \mathrm{~A}$, was mediated by aberrant methylation of the $\mathrm{CpG}$ island in glioma. A previous 

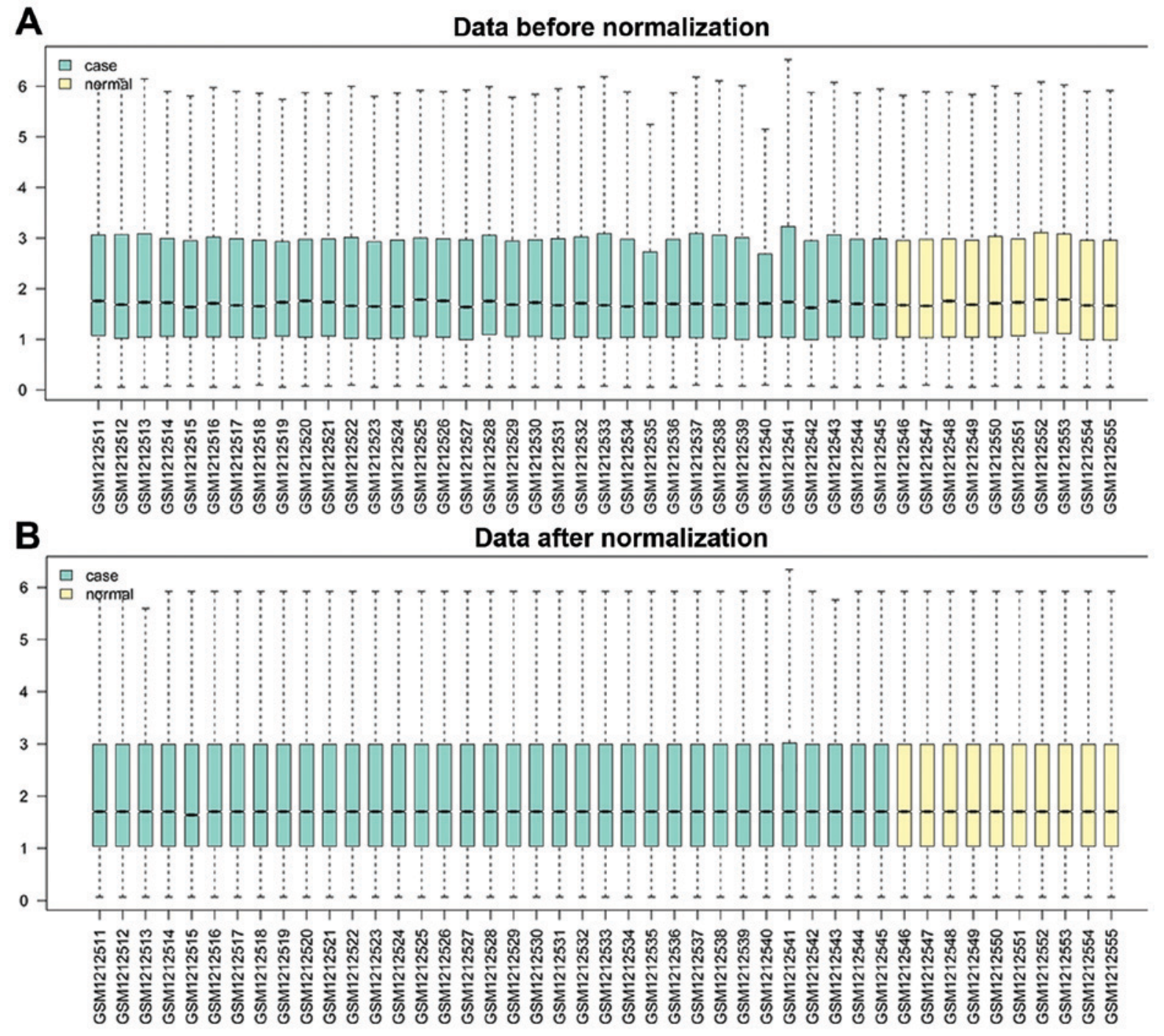

Figure 1. Expression boxplots for glioma-related genes. Box plots represent the mean \pm interquartile range, with whiskers extending to the minimum and maximum value. (A) Data prior to normalization; (B) data after normalization. GSM1212511-GSM1212545 (green) represent gliomas samples; GSM1212546-GSM1212555 (yellow) represent normal samples.

study also demonstrated that promoter methylation of DNA repair genes is associated with the tumorigenesis and development of glioma (15). However, despite extensive research, the exact mechanisms underlying how DNA methylation induces glioma remain unclear.

The present study compared DNA methylation and differentially expressed genes (DEGs) between glioma and normal tissues using Gene Expression Omnibus (GEO) data. Subsequently, the important genes and pathways in glioma were screened by enrichment analysis of transcriptional regulatory networks and sub-pathways. The present study aimed to determine the possible molecular mechanisms and the aberrant promoter methylation of tumor-associated genes in glioma, via identification of key genes and pathways. The results may provide a theoretical basis and identify potential molecular biological therapies for the treatment of glioma in children.

\section{Materials and methods}

Data sources. GSE50021 DNA microarray data (16) for gene expression profiling and GSE50022 dual-channel
DNA methylation data (16) were downloaded from GEO (http://www.ncbi.nlm.nih.gov/geo/). GSE50021 contains 45 samples, including 35 samples from patients with glioma (average age $1.008 \pm 1.190491$ years) and 10 autopsy samples of normal human brain tissue. Data from the chip (Illumina HumanHT-12 WG-DASL v4.0 Expression Beadchip; Illumina, San Diego, CA, USA) were annotated based on the GPL13938 platform. GSE50022 contains 28 samples from patients with glioma (average age, $0.9433333 \pm 0.781596$ years). Data from the chip (Illumina HumanMethylation450 BeadChip [UBC enhanced annotation v1.0]; Illumina) were annotated based on the GPL16304 platform.

Preprocessing of gene expression profiles. The expression values for genes with various capture probes were used to determine a mean value for each gene. The K-nearest neighbour (KNN) impute missing values module was used to impute missing values by assigning gene expression values based on the nearest neighbors of the gene $(k=10)$. All expression values were normalized using quantile normalization, as implemented 
Table I. Key methylation sites.

\begin{tabular}{|c|c|c|c|c|c|c|c|}
\hline ID & Chromosome & Mapinfo & tfbs_start & tfbs_end & $\begin{array}{l}\text { Transcription } \\
\text { factor }\end{array}$ & $\begin{array}{l}\text { Distance_- } \\
\text { closest_TSS }\end{array}$ & $\begin{array}{c}\text { Closest_TSS_ } \\
\text { gene_name }\end{array}$ \\
\hline cg06191091 & $\operatorname{chr} 17$ & 30583855 & 30583848 & 30583862 & USF & -9339 & RHBDL3 \\
\hline cg02629157 & $\operatorname{chr} 9$ & 138670609 & 138670546 & 138670568 & TCF11 & 25013 & KCNT1 \\
\hline $\operatorname{cg} 11709150$ & chr1 & 2440438 & 2440431 & 2440444 & TCF11 & 10256 & PLCH2 \\
\hline cg04585209 & chr11 & 6292311 & 6292257 & 6292272 & TAXCREB & 306 & CCKBR \\
\hline cg10707626 & chr3 & 51747098 & 51747027 & 51747051 & STAT5A & 6018 & GRM2 \\
\hline cg06191091 & $\operatorname{chr} 17$ & 30583855 & 30583849 & 30583860 & SREBP1 & -9339 & RHBDL3 \\
\hline $\operatorname{cg} 12603173$ & chr11 & 64508421 & 64508409 & 64508423 & RREB1 & -66 & RASGRP2 \\
\hline $\operatorname{cg} 11025960$ & chr3 & 51749188 & 51749177 & 51749195 & RFX1 & 8108 & GRM2 \\
\hline cg10692302 & $\operatorname{chr} 3$ & 51747227 & 51747224 & 51747245 & PPARG & 6147 & GRM2 \\
\hline cg02629157 & chr9 & 138670609 & 138670558 & 138670569 & POU6F1 & 25013 & KCNT1 \\
\hline $\operatorname{cg} 11014582$ & chr6 & 76333727 & 76333675 & 76333696 & PAX6 & -852 & LMO7 \\
\hline $\operatorname{cg} 04341461$ & chr1 & 2410006 & 2409978 & 2410006 & PAX5 & -1616 & PLCH2 \\
\hline $\operatorname{cg} 10692302$ & $\operatorname{chr} 3$ & 51747227 & 51747222 & 51747252 & PAX4 & 6147 & GRM2 \\
\hline $\operatorname{cg} 04625615$ & chr15 & 41788368 & 41788310 & 41788330 & P53 & 2313 & ITPKA \\
\hline cg07200386 & chr8 & 22079169 & 22079113 & 22079135 & OLF1 & 10682 & PHYHIP \\
\hline $\operatorname{cg} 11014582$ & chr6 & 76333727 & 76333676 & 76333683 & NKX25 & -852 & LMO7 \\
\hline cg09864712 & chr16 & 726786 & 726720 & 726749 & MYOGNF1 & 712 & RHBDL1 \\
\hline cg06191091 & $\operatorname{chr} 17$ & 30583855 & 30583848 & 30583862 & MYCMAX & -9339 & RHBDL3 \\
\hline cg00810908 & $\operatorname{chr} 3$ & 13612319 & 13612306 & 13612320 & MEIS1AHOXA9 & 2080 & FBLN2 \\
\hline $\operatorname{cg} 11025960$ & $\operatorname{chr} 3$ & 51749188 & 51749181 & 51749190 & LMO2COM & 8108 & GRM2 \\
\hline $\operatorname{cg} 03358506$ & chr8 & 22058702 & 22058688 & 22058703 & ISRE & 31149 & PHYHIP \\
\hline cg07776629 & chr16 & 57989122 & 57989116 & 57989129 & IRF2 & 15898 & CNGB1 \\
\hline cg07776629 & chr16 & 57989122 & 57989116 & 57989129 & IRF1 & 15898 & CNGB1 \\
\hline $\operatorname{cg} 10692302$ & $\operatorname{chr} 3$ & 51747227 & 51747225 & 51747244 & HNF4 & 6147 & GRM2 \\
\hline cg06632557 & chr11 & 61313548 & 61313495 & 61313505 & HMX1 & -3678 & SYT7 \\
\hline cg00155846 & $\operatorname{chr} 9$ & 138011566 & 138011506 & 138011522 & HAND1E47 & 14081 & OLFM1 \\
\hline $\operatorname{cg} 11025960$ & $\operatorname{chr} 3$ & 51749188 & 51749181 & 51749190 & GATA3 & 8108 & GRM2 \\
\hline $\operatorname{cg} 11025960$ & chr3 & 51749188 & 51749179 & 51749193 & GATA1 & 8108 & GRM2 \\
\hline $\operatorname{cg} 04625615$ & chr15 & 41788368 & 41788310 & 41788321 & GATA3 & 2313 & ITPKA \\
\hline cg05392169 & chr9 & 138011814 & 138011802 & 138011816 & FOXO3 & 14329 & OLFM1 \\
\hline
\end{tabular}

tfbs, transcription factor binding sites; TSS, transcription start site.

in the normalize.quantiles function of the preprocessCore $\mathrm{R}$ package (17). Expression values were displayed using a box plot.

Preprocessing of DNA methylation data. For probes with missing values in the original methylation index matrix the KNN impute missing values module was used to impute the missing values by assigning methylation gene expression values based on the nearest neighbors of the gene $(k=10)$.

Screening of differentially expressed genes (DEGs). The probe identifiers were converted into gene names. The DEG analysis was conducted using limma package (http://www.bioconductor.org). $\mid \log 2 \mathrm{FCl}>1$ and $\mathrm{P}<0.05$ were set as the cut-offs to screen for DEGs. DEGs were initially identified following an analysis using Student's t-test. The identified DEGs were considered glioma-related genes in children.
Screening of key methylated sites. According to the annotation information, probes were screened to determine which methylated sites were located near DEGs. Subsequently, the preliminarily reserved methylated sites were screened, and $\geq 80 \%$ of samples with a methylation index of $\geq 0.8$ were reserved. Due to the lack of clear definition of the promoter regions of genes, $50 \mathrm{~kb}$ on either side of the transcription start site was considered the promoter region. Finally, the methylated sites were re-screened, and methylated sites were reserved that were located $50 \mathrm{~kb}$ on either side of the transcription start site. After screening three times, the remaining methylated sites were considered key methylated sites, which were located in the promoter region of genes and have a high methylation index, thus potentially influencing the expression of downstream genes.

Screening of significant transcription factors. Using the transcription factor binding sites (TFBS) prediction tool from 

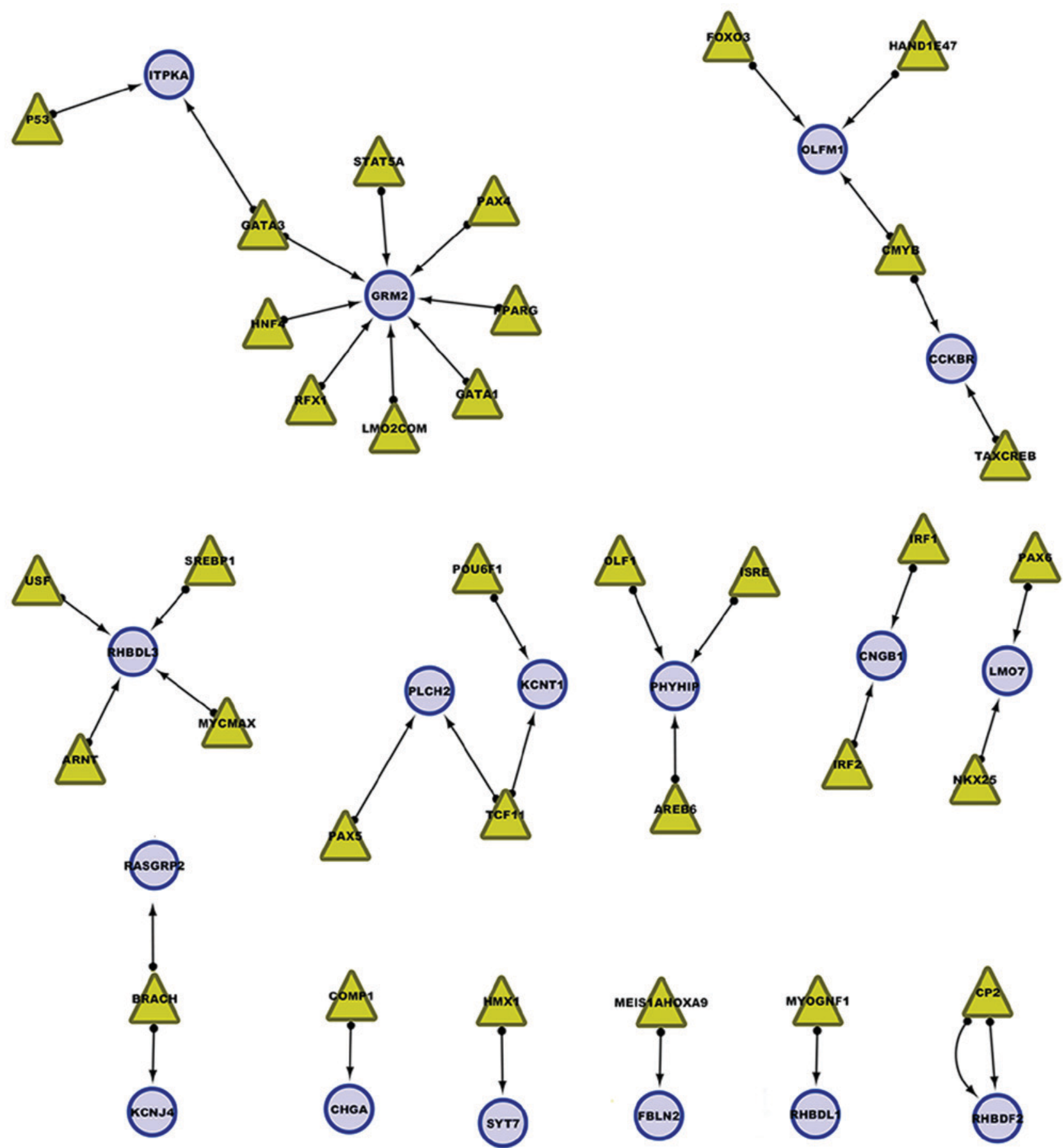

Figure 2. Transcriptional regulatory networks. Yellow triangles represent transcription factors; purple circles represent target genes; arrows represent transcriptional regulation.

University of California, Santa Cruz (UCSC; http://genome. ucsc.edu) the methylated sites were screened in TFBS. A transcription factor regulatory network was generated using Cytoscape (http://cytoscape.org/), which is an open source desktop tool for integrating, visualizing and analyzing data in the context of biological networks. Enrichment analysis of potential downregulated genes influenced by methylation was performed using the Database for Annotation, Visualization and Integrated Discovery (http://david.abcc.ncifcrf.gov/).

Analysis of risk sub-pathways. A hypergeometric test for risk sub-pathways was performed by application of the $\mathrm{R}$ package iSubpathwayMiner (http://cran.r-project.org/web/packages/iSubpathwayMiner/).

All genes that were mapped to sub-pathways with a significant enrichment were reported. These pathways were used to identify glioma-related pathways.

\section{Results}

Preprocessing of gene expression profiles and DNA methylation data. To explore glioma-related genes in children, 19,252 genes were identified from 45 samples following preprocessing of gene expression profiles. As shown in Fig. 1 expression boxplots were 


\section{path:00562_1 Inositol phosphate metabolism}

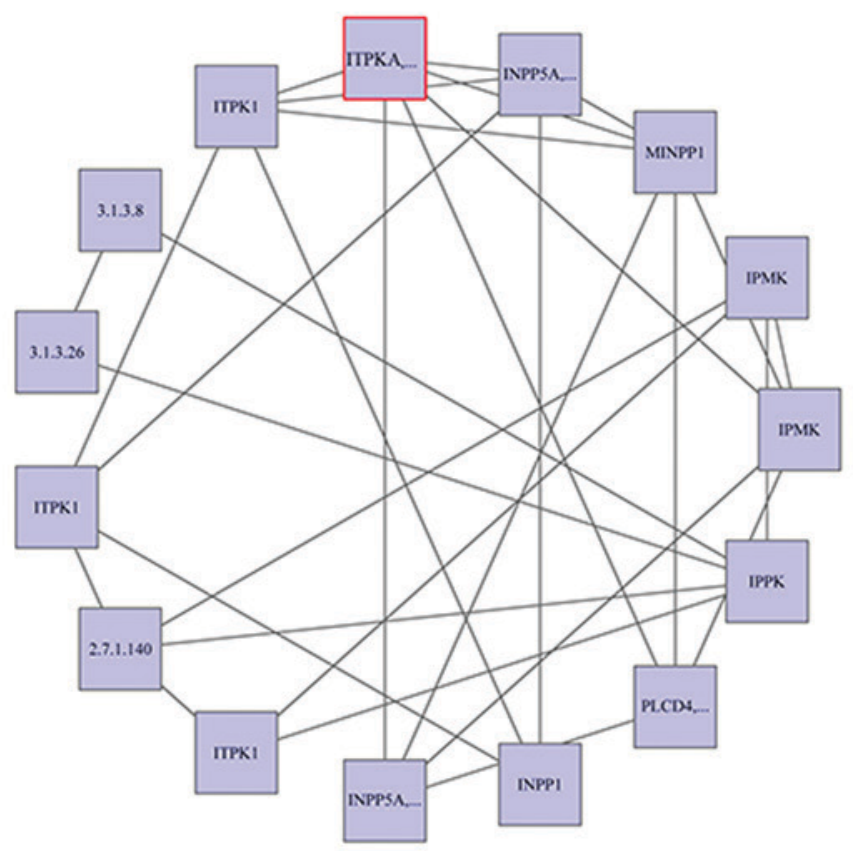

$p$-value $=0.0182007558864037$

\section{path:00562_3 Inositol phosphate metabolism}

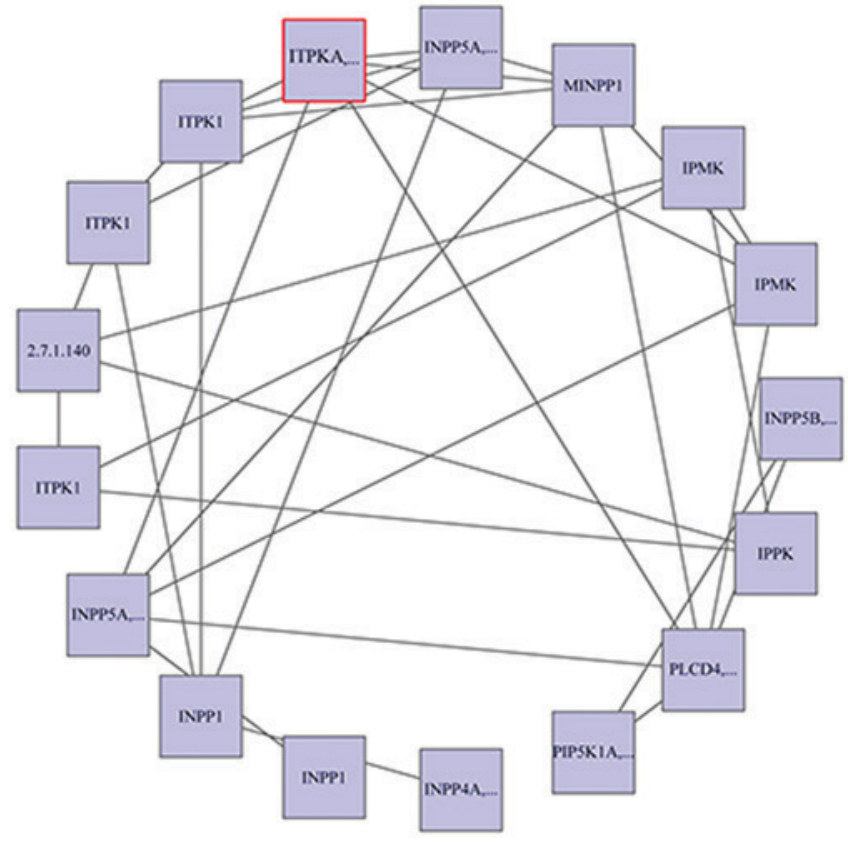

$\mathrm{p}$-value $=0.0280765742267587$

\section{path:00562_2 Inositol phosphate metabolism}

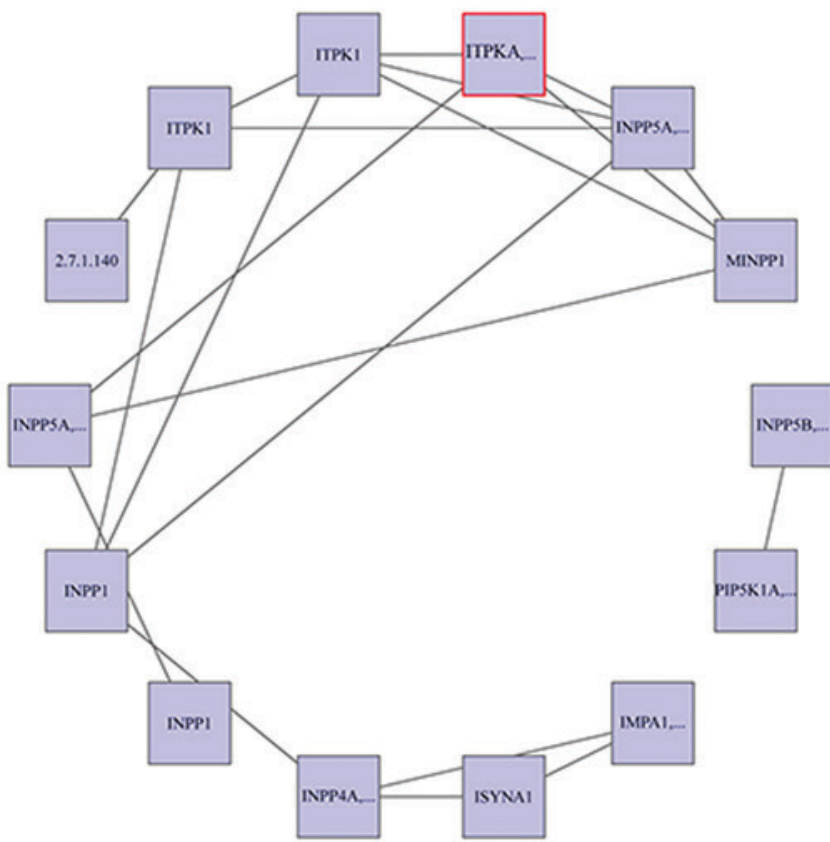

$p$-value $=0.0209035740380185$

\section{path:00562_4 Inositol phosphate metabolism}

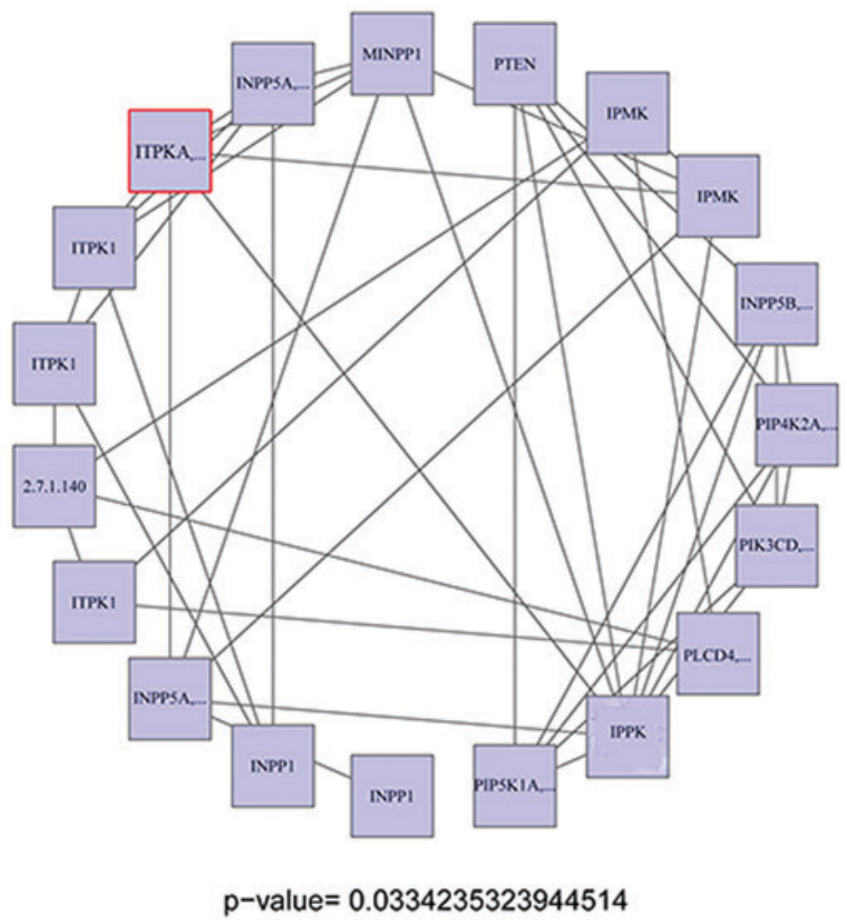

Figure 3. Risk sub-pathways in inositol phosphate metabolism. ITPKA gene is marked with a red rim. ITPKA, inositol-trisphosphate 3-kinase.

generated before and after normalization. The original expression datasets from all conditions were processed into expression estimates using the median polish algorithm. As shown in a box plot (Fig. 1B), the median of different samples was almost on the same line after normalization, which shows an excellent degree of standardization.

Data located in the $\mathrm{X} / \mathrm{Y}$ and mitochondrial chromosomes were removed. Subsequently, a methylation index array 
identified 371,920 sites from the 28 samples, following imputation of missing values.

Screening of DEGs. DEGs were analyzed using limma package. Using the selective conditions ( $\mid \log 2 \mathrm{FCl}>1$ and $\mathrm{P}<0.05), 58$ DEGs were identified, all of which were downregulated.

Identification of DNA methylation in the promoter regions of downregulated genes. TFBS methylation has a strong impact on the regulatory role of transcription factors and the normal expression of downstream genes, thus affecting body functioning. DNA methylation in the promoter region is an important aspect of DNA methylation in cancer $(18,19)$.

The 58 downregulated DEGs identified in the present study were overlapped with DNA methylation data; 1,123 methylation sites were revealed to be associated with the 58 downregulated genes. The sites were distributed evenly over the 22 autosomes. The methylation index of the 1,123 methylation sites was analyzed, and $\geq 80 \%$ of samples with a methylation index of $\geq 0.8$ were reserved. This resulted in the reservation of 168 methylation sites. To further analyze the 168 methylation sites, methylated sites located $50 \mathrm{~kb}$ on either side of the transcription start sites were reserved. This resulted in the reservation of 119 methylation sites located on the transcription start sites of 43 genes. These 43 genes are considered potential glioma susceptibility genes in children. Finally, 17 methylation sites located on 33 TFBS were identifed, which may regulate 17 of the downregulated target genes. These results were determined using the TFBS prediction tool from UCSC (Table I).

Analysis of transcriptional regulatory networks. To construct a transcription factor regulatory network, predictive models of transcriptional regulatory networks were generated using Cytoscape. A transcriptional regulatory network including 33 transcription factors and target genes is presented in Fig. 2. The eight methylation sites in the TFBS of glutamate metabotropic receptor 2 (GRM2) potentially influenced the binding of eight transcription factors to TFBS. It may therefore be hypothesized that the downregulation of GRM2 is caused by methylation of these TFBS. In addition, methylation sites in the TFBS of rhomboid, veinlet-like 3 (Drosophila) (RHBDL3) resulted in the failure of four transcription factors binding with TFBS, thus affecting transcriptional regulation. Furthermore, the transcription factor $\mathrm{CP} 2$ regulates rhomboid 5 homolog 2 (Drosophila) (RHBDF2) expression; in the present study, two methylation sites were detected in TFBS of CP2, which may influence transcriptional regulation of RHBDF2.

Functional analysis of key genes. To generate the downregulated target gene-related risk sub-pathways, a hypergeometric test was performed using R package iSubpathwayMiner. Four sub-pathways were generated, which were associated with inositol phosphate metabolism (hsa00562). As shown in Fig. 3 the inositol-trisphosphate 3-kinase protein (EC 2.7.1.127), which is coded by the ITPKA gene (marked with a red rim), appeared in all of the risk sub-pathways. In conclusion, ITPKA was considered to be the gene most associated with the risk of glioma in children.

\section{Discussion}

Bioinformatics is an interdisciplinary field that develops methods and software tools for understanding biological data. The identification of candidate genes and single nucleotide polymorphisms are common uses of bioinformatics to understand the genetic basis of disease (20). The present study identified 17 methylation sites located on 33 TFBS, which may regulate the 17 downregulated target genes. GRM2 was identified as one of the 17 downregulated target genes. Furthermore, ITPKA was revealed to be the gene most associated with glioma risk in children. The protein coded by the ITPKA gene appeared in all risk sub-pathways, thus suggesting that ITPKA is the gene most associated with glioma risk, and inositol phosphate metabolism is the key pathway associated with glioma in children.

Glioma is the most common type of primary brain tumor, which is associated with a poor prognosis for patients due to its aggressive growth behavior and highly invasive nature (21). Abnormal metabolic networks are closely associated with the pathogenesis of glioma. A metabolic pathway is a series of chemical reactions, which are catalyzed by enzymes, where the product of one enzyme acts as a substrate for the next. Abnormal gene expression of specific enzymes may affect other enzymatic genes in the network. Therefore, identification of disease-related genes in the metabolic pathway is of great importance.

The correct targeting, localization, regulation and signaling of metabotropic glutamate receptor 2 (mGluR2), which is coded by the GRM2 gene, represents a major mechanism underlying the complex function of neuronal networks (22). Dysfunction of mGluR and associated proteins is associated with neurodegenerative and neuropsychiatric disorders. Several types of glioma have been reported to release high levels of glutamate, which promotes malignancy. A previous study reported that activation of mGluR2 supports the growth of human glioma cells in culture, and that antagonists of mGluR2 should be assessed for their ability to reduce tumour growth in vivo (23). Arcella et al (24) demonstrated that $\mathrm{mGlu} 2 / 3$ receptor antagonists are of potential use in the experimental treatment of malignant gliomas. Furthermore, ectopic expression of ITPKA in lung cancer cells has been shown to increase their metastatic potential, since the protein exhibits two actin-regulating activities (25). The ITPKA gene has also been reported to be hypermethylated in human patients with normal karyotype acute myeloid leukemia (26). Furthermore, the remaining 15 downregulated genes identified in the present study may be beneficial for the generation of a target therapy for the treatment of patients with glioma.

In conclusion, the present study proposed a novel method for extracting DEGs from gene expression datasets combined with DNA methylation data, and applied it to the occurrence of glioma in children. Results of the functional analysis revealed that ITPKA was considered the gene most associated with the risk of glioma, and inositol phosphate metabolism may serve an important role in glioma in children. The identification of specific genes may improve understanding regarding the pathogenesis and possible therapeutic targets of glioma in children. 


\section{References}

1. Brat DJ, Scheithauer BW, Fuller GN and Tihan T: Newly codified glial neoplasms of the 2007 WHO classification of tumours of the central nervous system: Angiocentric glioma, pilomyxoid astrocytoma and pituicytoma. Brain Pathol 17: 319-324, 2007.

2. Osoba D, Brada M, Prados MD and Yung WK: Effect of disease burden on health-related quality of life in patients with malignant gliomas. Neuro Oncol 2: 221-228, 2000.

3. Stupp R, Hegi ME, Mason WP, van den Bent MJ, Taphoorn MJ, Janzer RC, Ludwin SK, Allgeier A, Fisher B, Belanger K, et al: Effects of radiotherapy with concomitant and adjuvant temozolomide versus radiotherapy alone on survival in glioblastoma in a randomised phase III study: 5-year analysis of the EORTC-NCIC trial. Lancet Oncol 10: 459-466, 2009.

4. Van Meir EG, Hadjipanayis CG, Norden AD, Shu HK, Wen PY and Olson JJ: Exciting new advances in neuro-oncology: The avenue to a cure for malignant glioma. CA Cancer J Clin 60 166-193, 2010.

5. Paul SP, Perrow R and Webster MA: Brain tumours in children: Reducing time to diagnosis. Emerg Nurse 22: 32-36; quiz 37, 2014.

6. Pollack IF: Brain tumors in children. N Engl J Med 331: 1500-1507, 1994.

7. Turner CD, Chordas CA, Liptak CC, Rey-Casserly C, Delaney BL, Ullrich NJ, Goumnerova LC, Scott RM, Begley HC, Fletcher WJ, et al: Medical, psychological, cognitive and educational late-effects in pediatric low-grade glioma survivors treated with surgery only. Pediatr Blood Cancer 53: 417-423, 2009.

8. Reimers TS, Mortensen EL and Schmiegelow K: Memory deficits in long-term survivors of childhood brain tumors may primarily reflect general cognitive dysfunctions. Pediatr Blood Cancer 48: 205-212, 2007.

9. Furnari FB, Fenton T, Bachoo RM, Mukasa A, Stommel JM, Stegh A, Hahn WC, Ligon KL, Louis DN, Brennan C, et al: Malignant astrocytic glioma: Genetics, biology, and paths to treatment. Genes Dev 21: 2683-2710, 2007.

10. Wu Ct and Morris JR: Genes, genetics, and epigenetics: A correspondence. Science 293: 1103-1105, 2001.

11. Portela A and Esteller M: Epigenetic modifications and human disease. Nat Biotechnol 28: 1057-1068, 2010.

12. Goll MG and Bestor TH: Eukaryotic cytosine methyltransferases. Annu Rev Biochem 74: 481-514, 2005.

13. Herman JG, Latif F, Weng Y, Lerman MI, Zbar B, Liu S, Samid D, Duan DS, Gnarra JR, Linehan WM, et al: Silencing of the VHL tumor-suppressor gene by DNA methylation in renal carcinoma. Proc Natl Acad Sci USA 91: 9700-9704, 1994.
14. Costello JF, Berger MS, Huang HS and Cavenee WK: Silencing of p16/CDKN2 expression in human gliomas by methylation and chromatin condensation. Cancer Res 56: 2405-2410, 1996.

15. Skiriute D, Vaitkiene P, Saferis V, Asmoniene V, Skauminas K, Deltuva VP and Tamasauskas A: MGMT, GATA6, CD81, DR4, and CASP8 gene promoter methylation in glioblastoma. BMC Cancer 12: 218, 2012.

16. Buczkowicz P, Hoeman C, Rakopoulos P, Pajovic S, Letourneau L, Dzamba M, Morrison A, Lewis P, Bouffet E, Bartels U, et al: Genomic analysis of diffuse intrinsic pontine gliomas identifies three molecular subgroups and recurrent activating ACVR1 mutations. Nat Genet 46: 451-456, 2014.

17. Bolstad BM: preprocessCore: A collection of pre-processing functions. R Package, Version 1.22.0, 2014.

18. Tekpli X, Landvik NE, Anmarkud KH, Skaug V, Haugen A and Zienolddiny S: DNA methylation at promoter regions of interleukin 1B, interleukin 6 , and interleukin 8 in non-small cell lung cancer. Cancer Immunol Immunother 62: 337-345, 2013.

19. Spiegl-Kreinecker S, Pirker C, Filipits M, Lötsch D, Buchroithner J, Pichler J, Silye R, Weis S, Micksche M, Fischer J and Berger W: O6-Methylguanine DNA methyltransferase protein expression in tumor cells predicts outcome of temozolomide therapy in glioblastoma patients. Neuro Oncol 12: 28-36, 2010.

20. Véron A, Blein S and Cox DG: Genome-wide association studies and the clinic: A focus on breast cancer. Biomark Med 8: 287-296, 2014

21. Kleihues P and Sobin LH: World Health Organization classification of tumors. Cancer 88: 2887, 2000.

22. Enz R: Metabotropic glutamate receptors and interacting proteins: Evolving drug targets. Curr Drug Targets 13: 145-156, 2012.

23. D'Onofrio M, Arcella A, Bruno V, Ngomba RT, Battaglia G, Lombari V, Ragona G, Calogero A and Nicoletti F: Pharmacological blockade of mGlu2/3 metabotropic glutamate receptors reduces cell proliferation in cultured human glioma cells. J Neurochem 84: 1288-1295, 2003.

24. Arcella A, Carpinelli G, Battaglia G, D'Onofrio M, Santoro F, Ngomba RT, Bruno V, Casolini P, Giangaspero F and Nicoletti F: Pharmacological blockade of group II metabotropic glutamate receptors reduces the growth of glioma cells in vivo. Neuro Oncol 7: 236-245, 2005.

25. SchröderD,Rehbach C,Seyffarth C,NeuenschwanderM,Kries JV and Windhorst S: Identification of a new membrane-permeable inhibitor against inositol-1,4,5-trisphosphate-3-kinase A. Biochem Biophys Res Commun 439: 228-234, 2013.

26. Sonnet M, Claus R, Becker N, Zucknick M, Petersen J, Lipka DB Oakes CC, Andrulis M, Lier A, Milsom MD, et al: Early aberrant DNA methylation events in a mouse model of acute myeloid leukemia. Genome Med 6: 34, 2014. 